\title{
Article
}

\section{Real-World Experience of the One-Year Efficacy of Rifaximin Add-On to Lactulose Is Superior to Lactulose Alone in Patients with Cirrhosis Complicated with Recurrent Hepatic Encephalopathy in Taiwan}

\author{
Ching Chang ${ }^{1}\left(\mathbb{D}\right.$, Chien-Hao Huang ${ }^{1,2}$, Hsiao-Jung Tseng ${ }^{3}$, Fang-Chen Yang ${ }^{1}$ and Rong-Nan Chien ${ }^{1,2, *(\mathbb{D})}$ \\ 1 Division of Hepatology, Department of Gastroenterology and Hepatology, Chang-Gung Memorial Hospital, \\ Linkou Medical Center, Taoyuan 33305, Taiwan; o78714@gmail.com (C.C.); \\ huangchianhou@gmail.com (C.-H.H.); whiteeva907@gmail.com (F.-C.Y.) \\ 2 College of Medicine, Chang-Gung University, Taoyuan 33305, Taiwan \\ 3 Biostatistics Unit, Clinical Trial Center, Chang Gung Memorial Hospital, Linkou Medical Center, \\ Taoyuan 33305, Taiwan; allebjht@gmail.com \\ * Correspondence: ronald@cgmh.org.tw; Tel.: +886-3-3281200 (ext. 8107); Fax: +886-3-3272236
}

check for updates

Citation: Chang, C.; Huang, C.-H.; Tseng, H.-J.; Yang, F.-C.; Chien, R.-N. Real-World Experience of the One-Year Efficacy of Rifaximin Add-On to Lactulose Is Superior to Lactulose Alone in Patients with Cirrhosis Complicated with Recurrent Hepatic Encephalopathy in Taiwan. J. Pers. Med. 2021, 11, 478. https://doi.org/10.3390/ jpm11060478

Academic Editors: Pritmohinder S. Gill and Frank Vinholt Schiødt

Received: 2 March 2021

Accepted: 21 May 2021

Published: 27 May 2021

Publisher's Note: MDPI stays neutral with regard to jurisdictional claims in published maps and institutional affiliations.

Copyright: () 2021 by the authors. Licensee MDPI, Basel, Switzerland. This article is an open access article distributed under the terms and conditions of the Creative Commons Attribution (CC BY) license (https:// creativecommons.org/licenses/by/ $4.0 /)$.

\begin{abstract}
Background: Hepatic encephalopathy (HE), a neuropsychiatric complication of decompensated cirrhosis, is associated with high mortality and high risk of recurrence. Rifaximin add-on to lactulose for 3 to 6 months is recommended for the prevention of recurrent episodes of HE after the second episode. However, whether the combination for more than 6 months is superior to lactulose alone in the maintenance of HE remission is less evident. Therefore, the aim of this study is to evaluate the one-year efficacy of rifaximin add-on to lactulose for the maintenance of HE remission in Taiwan. Methods: We conducted a real-world single-center retrospective cohort study to compare the long-term efficacy of rifaximin add-on to lactulose (group $\mathrm{R}+\mathrm{L}$ ) versus lactulose alone (group $\mathrm{L}$, control group). Furthermore, the treatment efficacy before and after rifaximin add-on to lactulose was also analyzed. The primary endpoint of our study was time to first HE recurrence (Conn score $\geq 2$ ). All patients were followed up every three months until death, and censored at one year if still alive. Results and Conclusions: 12 patients were enrolled in group $\mathrm{R}+\mathrm{L}$. Another 31 patients were stratified into group L. Sex, comorbidity, ammonia level, and ascites grade were matched while age, HE grade, and model for end-stage liver disease (MELD) score were adjusted in the multivariable logistic regression model. Compared with group L, significant improvement in the maintenance of HE remission and decreased episodes and days of HE-related hospitalizations were demonstrated in group $\mathrm{R}+\mathrm{L}$. The serum ammonia levels were significantly lower at the 3rd and 6th month in group 1. Concerning changes before and after rifaximin add-on in group $\mathrm{R}+\mathrm{L}$, mini-mental status examination (MMSE), episodes of hospitalization, and variceal bleeding also improved at 6 and 12 months. Days of hospitalization, serum ammonia levels also improved at 6 th month. Except for concern over price, no patients discontinued rifaximin due to adverse events or complications. The above results provide evidence for the one-year use of rifaximin add-on to lactulose in reducing HE recurrence and HE-related hospitalization for patients with decompensated cirrhosis.
\end{abstract}

Keywords: rifaximin; lactulose; cirrhosis; hepatic encephalopathy; Taiwan

\section{Introduction}

Hepatic encephalopathy (HE), a neuropsychiatric complication of decompensated cirrhosis, clinically manifests from minimal cognitive dysfunction to lethargy and, most seriously, coma [1]. HE is associated with high mortality and high risk of recurrence [1,2] and has a major impact on health-related quality of life in patients with liver cirrhosis [3]. 
There are various treatment choices for HE, including nonabsorbable disaccharides, antibiotics, and other potential therapies such as probiotics, branched-chain amino acids, and glutaminase inhibitors employing various mechanisms [1].

Lactulose, a nonabsorbable disaccharide, decreases the absorption of nitrogen-containing substances from the gastrointestinal tract via cathartic effects and reduces ammonia burden through converting ammonia to nonabsorbable ammonium by changing colonic $\mathrm{pH}$ [4]. The effect of lactulose for preventing overt HE is well reported [5], but adverse effects like severe diarrhea, bloating, flatulence, nausea, and vomiting may lower medication compliance [6].

Rifaximin, a poorly absorbed antibiotic, was approved in 2010 by the United States food drug administration (US FDA) for the treatment of overt hepatic encephalopathy via decreasing ammonia-generating enteric bacteria $[7,8]$. Its effects of reducing ammonia levels, preventing overt HE recurrence, as well as lowering HE-related hospitalization are documented in previous studies [9-11]. According to the current practice guideline by American association for the study of liver disease (AASLD)/European association for the study of liver (EASL), rifaximin add-on to lactulose over 3 to 6 months is recommended for the prevention of recurrent episodes of HE after the second episode [1]. Furthermore, rifaximin is reported to be safe for long-term use without obvious side effects due to characteristic of minimal absorption and fewer systemic effects $[9,11]$.

However, the superiority of combination rifaximin and lactulose for more than six month over lactulose monotherapy alone in the maintenance of HE remission is less evident [12-14]. A few studies have investigated the prolonged effects of HE remission from a combination use of rifaximin with lactulose [13,15]. One study compared rifaximin with a placebo group rather than a lactulose group [16]. In addition, the use of rifaximin for the treatment of HE is less common in Taiwan because lactulose is supported by national health insurance administration while rifaximin is not.

Therefore, the aim of this study is to evaluate whether the one-year efficacy of rifaximin add-on to lactulose therapy is superior to lactulose alone for the maintenance of remission from overt hepatic encephalopathy in cirrhotic patients in Taiwan by using real-world data.

\section{Materials and Methods}

\subsection{Study Design}

We conducted a single-center retrospective cohort study to compare the long-term efficacy of rifaximin add-on to lactulose versus lactulose alone. In addition, the treatment efficacy regarding HE before and after rifaximin add-on to lactulose was also analyzed. This study was approved by the Institutional Review Board of Linkou Chang Gung Memorial Hospital, and informed consent was obtained from all patients in our study (201701810B0).

\subsection{Patient Selection}

From January 2015 to December 2019, consecutive patients age 18 years or older and diagnosed with liver cirrhosis complicated by HE (at least two episodes during the previous 6 months) were included. Patients with other diagnosed neurological or psychiatric comorbidities (like Alzheimer's, Parkinson's, stroke with neurological deficit), advanced age with dementia, alcoholism-related brain dysfunction (such as Wernicke encephalopathy, Korsakoff syndrome), active use of alcohol/opioids/other substance abuse, use of antibiotics/probiotics/anti-motility drugs, inability to take oral medication, unstable vital signs, transjugular intrahepatic portosystemic shunt (TIPS) or surgical shunts, liver transplantation within one-year follow-up, non-curative hepatocellular carcinoma (HCC) ( $\geq$ Barcelona clinic liver cancer classification (BCLC) stage B), or active extra-hepatic malignancy were excluded.

The indications of rifaximin use in our study were persistent recurrent HE (at least two episodes within 6 months) under lactulose therapy or intolerance to lactulose due to adverse effects such as diarrhea and bloating. 
Patients were enrolled for group $\mathrm{R}+\mathrm{L}$ upon satisfying the above criteria and if financially feasible for the patients (cost: about USD 7600/year). All patients who received rifaximin $550 \mathrm{mg}$ twice daily continuously took lactulose as a combination treatment, and lactulose dosage was adjusted to reduce intolerance. Unless severe adverse effects or economic burden occurred, there was no restriction on the use of rifaximin in our study.

On the other hand, patients receiving only lactulose (30 to $45 \mathrm{~mL}$ twice to four times daily) were selected as the control group (group L).

\subsection{Data Collection}

Baseline clinical parameters such as age, sex, ascites status, prior HE grade, esophageal varices (EV) status, EV bleeding (EVB) episodes, HCC, hepatorenal syndrome (HRS), spontaneous bacterial peritonitis (SBP), and comorbidities (diabetes mellitus (DM), end stage renal disease (ESRD), heart failure) were documented from medical records. The presence and severity (nil, mild, moderate, severe) of ascites were assessed by abdominal ultrasonography. Esophagogastroduodenoscopy was used for the evaluation of esophageal/gastric varices.

We also collected laboratory data, including ammonia levels, white blood cell (WBC) count, hemoglobin $(\mathrm{Hb})$ level, platelet (PLT) count, prothrombin time/INR, creatinine, aspartate aminotransferase (AST), alanine aminotransferase (ALT), total bilirubin, albumin, ammonia, and model for end-stage liver disease (MELD) scores, before therapy and during follow-up.

Patients visited our outpatient clinic every 3 months, with evaluation of clinical conditions including mini-mental state examination (MMSE) and psychometric hepatic encephalopathy score (PHES) tests for cognitive function, biochemistry data, ascites condition, and any adverse events or complications.

\subsection{Treatment Efficacy and Safety Assessments}

Treatment efficacy comprises primary and secondary endpoints. The primary endpoint of our study was time to first HE recurrence (Conn score $\geq 2$ ) within one year and was compared between the combination group $(\mathrm{R}+\mathrm{L})$ and the lactulose monotherapy group (L). The secondary endpoints included the numbers and days of hospitalization attributed to $\mathrm{HE}$, the serum ammonia level during follow-up, the cognition function (MMSE), serum ammonia level, renal function, and varices condition, compared between the combination and lactulose monotherapy groups every three months up to one year. In addition, these clinical and laboratory parameters, including the cognition function MMSE score, were also compared before and after rifaximin therapy within the rifaximin add-on group.

For safety evaluation of long-term rifaximin use, we documented any discomfort, adverse events, or complications if present at follow-up.

\subsection{Statistical Analysis}

Continuous variables are expressed as median [interquartile range (IQR)]. A nonparametric Mann-Whitney U test was used to compare the continuous variables between group R + L vs. group L. Categorical variables are described as frequencies and percentages with the Chi-square test for comparison. When it came to a situation where more than $20 \%$ of data cells presented an expected frequency of $<5$, Fisher's exact test was substituted for the Chi-square test. Univariable and multivariable logistic regression analysis were performed for adjusting predictors for HE recurrence within 12 months. Kaplan-Meier and Log-rank test were used for univariable survival analysis of the efficacy of group $R$ $+\mathrm{L}$ vs. group L in preventing HE recurrence within 12 months in patients with cirrhosis complicated by HE. As for comparing the clinical parameters at baseline and after rifaximin add-on to lactulose initiated in group $\mathrm{R}+\mathrm{L}$ patients at every visit, repeated measurement ANOVA was used. However, for clarity and simplicity, only the baseline, 6 th and 12th month data comparisons were demonstrated. Statistics were performed using SPSS software (SPSS Inc., Chicago, IL, USA). A $p$ value of $<0.05$ was considered statistically significant. Power analysis was also performed. 


\section{Results}

\subsection{Flowchart and Patient Characteristics}

As shown in Figure 1, 60 patients fulfilling the inclusion criteria were included. After excluding 17 patients who met the exclusion criteria, a total of 12 patients received rifaximin add-on to lactulose combination therapy (group $\mathrm{R}+\mathrm{L}$ ), and 31 patients who received lactulose monotherapy (group L) were enrolled and analyzed. Demographic and baseline characteristics are shown in Table 1. Except for age, HCC status, and MELD score, there were no statistical difference between the two groups for sex, HE grade, ascites amount, EV grade, EVB, SBP, HRS, DM, ESRD, heart failure, serum ammonia, INR, WBC, $\mathrm{Hb}, \mathrm{PLT}$, creatinine, bilirubin total, AST/ALT, or albumin.

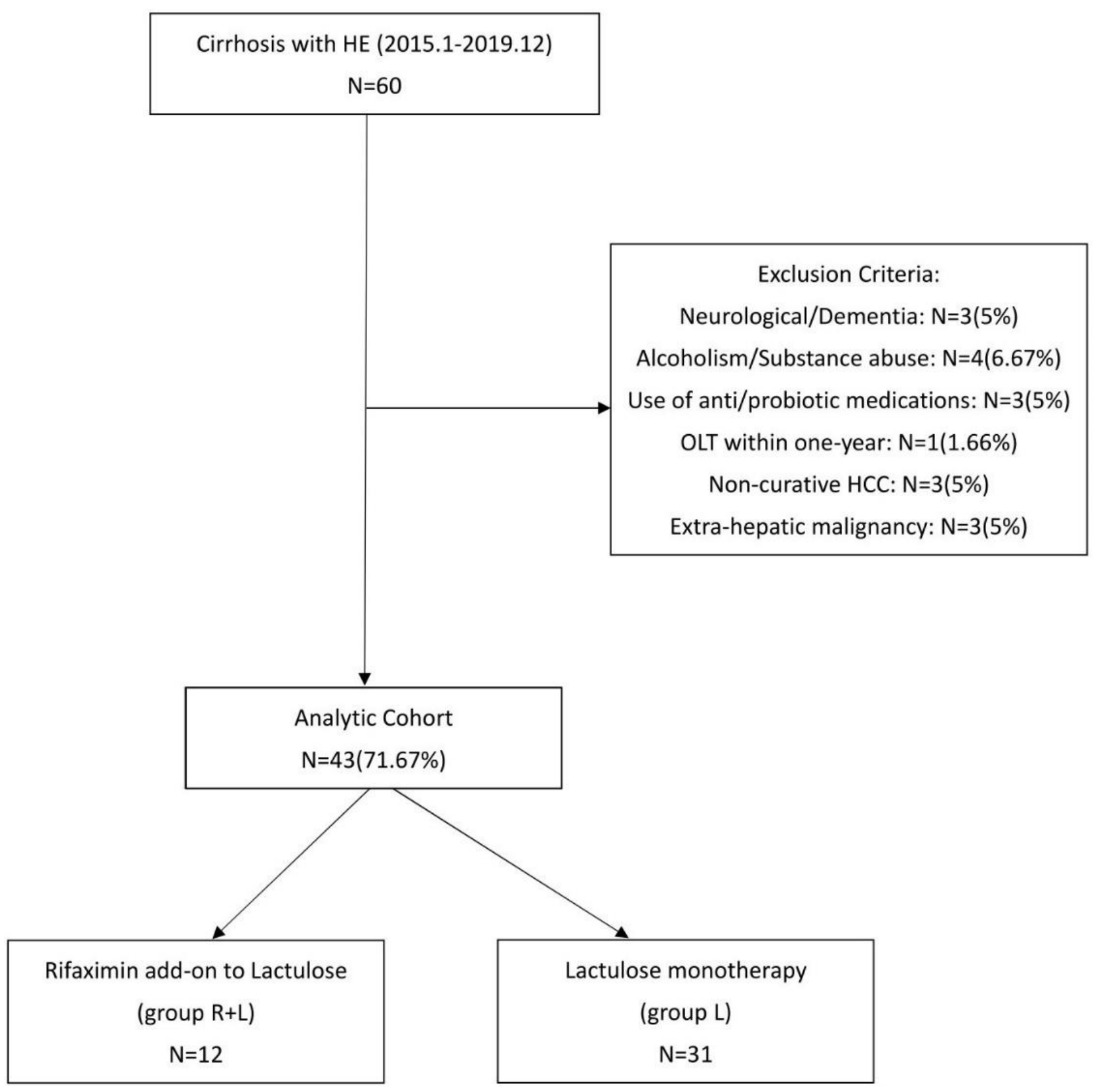

Figure 1. Flow chart of the study. 
Table 1. Demography and baseline clinical characteristics of group 1 and group 2 patients.

\begin{tabular}{|c|c|c|c|}
\hline & Group 1 Rifaximin + Lactulose & Group 2 Lactulose & Voln \\
\hline Baseline Parameter & $\mathrm{N}=12$ & $\mathbf{N}=31$ & $p$-value \\
\hline \multicolumn{4}{|l|}{ Clinical parameters } \\
\hline Age, mean $\pm S D$ & $67 \pm 7.95$ & $57.58 \pm 12.28$ & 0.007 \\
\hline Males (\%) & $6(50 \%)$ & $20(64.5 \%)$ & 0.388 \\
\hline HE grade $\$$ & & & 0.160 \\
\hline Minimal/I/II/III/IV No. & $4 / 2 / 4 / 2 / 0$ & $0 / 6 / 15 / 9 / 1$ & \\
\hline Ascites amount & & & 0.210 \\
\hline Nil/Mild/Moderate/Severe No. & $7 / 2 / 2 / 1$ & $13 / 4 / 6 / 8$ & \\
\hline EV & & & 0.052 \\
\hline Nil/F1/F2/F3 No. & $1 / 3 / 3 / 5$ & $10 / 8 / 8 / 5$ & \\
\hline EVB No. (\%) & $6(50 \%)$ & $7(22.6 \%)$ & 0.108 \\
\hline SBP No. (\%) & $0(0 \%)$ & $0(0 \%)$ & \\
\hline HRS No. $(\%)$ & $0(0 \%)$ & $0(0 \%)$ & \\
\hline HCC No. $(\%)$ & $4(33.3 \%)$ & $0(0 \%)$ & $<0.001$ \\
\hline DM No. $(\%)$ & $5(41.7 \%)$ & $7(22.5 \%)$ & 0.216 \\
\hline ESRD No. $(\%)$ & $1(8.3 \%)$ & $5(16.1 \%)$ & 0.513 \\
\hline Heart Failure No. (\%) & $1(8.3 \%)$ & $1(3.2 \%)$ & 0.481 \\
\hline \multirow{2}{*}{\multicolumn{4}{|c|}{$\begin{array}{l}\text { Laboratory parameters } \\
\text { Median (IQR) }\end{array}$}} \\
\hline & & & \\
\hline Ammonia $(\mu \mathrm{g} / \mathrm{dL})$ & $226.0(186.40-262.0)$ & $224.0(174.75-317.0)$ & 0.895 \\
\hline MELD & $14.05(11.87-16.55)$ & $17.0(14.0-22.0)$ & 0.048 \\
\hline INR & $1.40(1.30-1.60)$ & $1.50(1.30-1.70)$ & 0.874 \\
\hline WBC $\left(10^{3} / \mathrm{uL}\right)$ & $3.45(2.75-5.37)$ & $4.85(3.50-6.35)$ & 0.060 \\
\hline Hemoglobin (g/dL) & $9.55(8.82-10.30)$ & $10.0(8.65-12.17)$ & 0.549 \\
\hline $\operatorname{PLT}\left(10^{3} / \mathrm{uL}\right)$ & $62.0(37.0-79.75)$ & $81.0(59.0-111.0)$ & 0.091 \\
\hline Creatinine $(\mathrm{mg} / \mathrm{dL})$ & $0.95(0.83-1.09)$ & $1.13(0.63-1.98)$ & 0.924 \\
\hline Bilirubin, Total (mg/dL) & $1.85(1.30-2.12)$ & $2.50(1.30-3.30)$ & 0.095 \\
\hline $\operatorname{AST}(\mathrm{U} / \mathrm{L})$ & $40.0(34.0-53.0)$ & $51.0(37.0-73.0)$ & 0.188 \\
\hline ALT (U/L) & $22.0(20.0-25.0)$ & $25.50(18.75-31.50)$ & 0.461 \\
\hline Albumin (g/dL) & $3.0(2.55-3.38)$ & $2.95(2.40-3.36)$ & 0.987 \\
\hline
\end{tabular}

$\$$ West-Heaven classification [Conn H.O., Liberathal M.M. The hepatic coma syndromes and lactulose. Williams and Wilkins 1979; 1-121]. [DOI:10.1016/0016-5085(79)90191-4]. EV: esophageal varices; F1: form 1; EVB: esophageal variceal bleeding; SBP: spontaneous bacterial peritonitis; HRS: hepatorenal syndrome; HCC: hepatocellular carcinoma; DM: diabetes mellitus; ESRD: end-stage-renal disease. MELD: model for end-stage liver disease; INR: international normalized ratio; WBC: white blood cells; PLT: platelet; AST: aspartate transaminase; ALT: alanine transaminase.

Regarding clinical parameters, the mean ages in group $\mathrm{R}+\mathrm{L}$ patients were older than that in group $\mathrm{L}$ patients ( 67 vs. 58 years-old, $p=0.007$ ). In group $\mathrm{R}+\mathrm{L}$, there were 4 patients diagnosed with minimal HE by PHES test, 6 with grade $1 \sim 2 \mathrm{HE}, 2$ with grade $3 \sim 4$ HE. In group L, there were 21 patients diagnosed with grade $1 \sim 2 \mathrm{HE}$, while 10 patients had grade 3 4 HE by West Haven classification. There were 4 patients diagnosed with HCC in group $\mathrm{R}+\mathrm{L}$ while 0 patients were diagnosed with HCC in group L. Regarding laboratory parameters, the median MELD score in group $\mathrm{R}+\mathrm{L}$ was lower than that in group L (14.05 vs. $17.00, p=0.048)$.

\subsection{Outcomes Analysis Between Group $R+L$ Versus Group L Primary Outcome}

Upon primary endpoint analysis, the median time to first $\mathrm{HE}$ recurrence (Conn score $\geq 2$ ) in the group $\mathrm{R}+\mathrm{L}$ was significantly longer than that in group $\mathrm{L}$ during follow-up (204.50 days, IQR: $170.25-492.00$ vs. 125.00 days, IQR: $42.25-247.00$. $p=0.044$ ). A multivariable logistic regression analysis was performed to adjust for those confounding factors. MELD score instead of Child Turcotte-Pugh (CTP) score was used in the regression model because CTP score possessed collinearity with HE grade. As shown in Table 2, independent to baseline HE grade, the odds ratio of HE recurrence within 1 year between two groups was 0.214 $(p=0.045)$, suggesting that there was a relatively lower risk of HE recurrence for patients in the group R + L compared to that in the group L. Kaplan-Meier estimates of the efficacy of 
group $\mathrm{R}+\mathrm{L}$ vs. group $\mathrm{L}$ in avoiding HE recurrence within 12 months were also consistent, as shown in Figure 2.

Table 2. Logistic regression estimates of baseline parameters for predicting HE recurrence within 1 year.

\begin{tabular}{|c|c|c|c|c|}
\hline \multirow[b]{2}{*}{ Variables } & \multicolumn{2}{|c|}{ Univariable Logistic Reg. } & \multicolumn{2}{|c|}{ Multivariable Logistic reg. } \\
\hline & OR $(95 \% \mathrm{CI})$ & $p$-Value & OR $(95 \% \mathrm{CI})$ & $p$-Value \\
\hline Group (rifaximin + lactulose vs. lactulose) & $0.148(0.032,0.694)$ & 0.015 & $0.214(0.037,0.925)$ & 0.045 \\
\hline Age & $0.985(0.927,1.047)$ & 0.628 & & \\
\hline Sex & $1.719(0.377,7.849)$ & 0.484 & & \\
\hline MELD & $1.160(0.969,1.389)$ & 0.105 & & \\
\hline $\begin{array}{c}\text { HE grade } \\
\text { (>II vs. I and minimal) }\end{array}$ & $13.067(2.5000,68.291)$ & 0.002 & $10.182(1.793,57.802)$ & 0.009 \\
\hline $\operatorname{EVB}(\mathrm{n}, \%)$ & $6(50 \%)$ & 0.832 & & \\
\hline HCC & $3.875(0.471,31.912)$ & 0.208 & & \\
\hline $\mathrm{DM}$ & $2.083(0.467,9.288)$ & 0.336 & & \\
\hline ESRD & $1.812(0.280,11.750)$ & 0.533 & & \\
\hline Heart Failure & $3.556(0.202,62.632)$ & 0.386 & & \\
\hline
\end{tabular}

MELD: model for end-stage liver disease; HE: hepatic encephalopathy; EVB: esophageal variceal bleeding; HCC: hepatocellular carcinoma; DM: diabetes mellitus; ESRD: end-stage-renal disease.

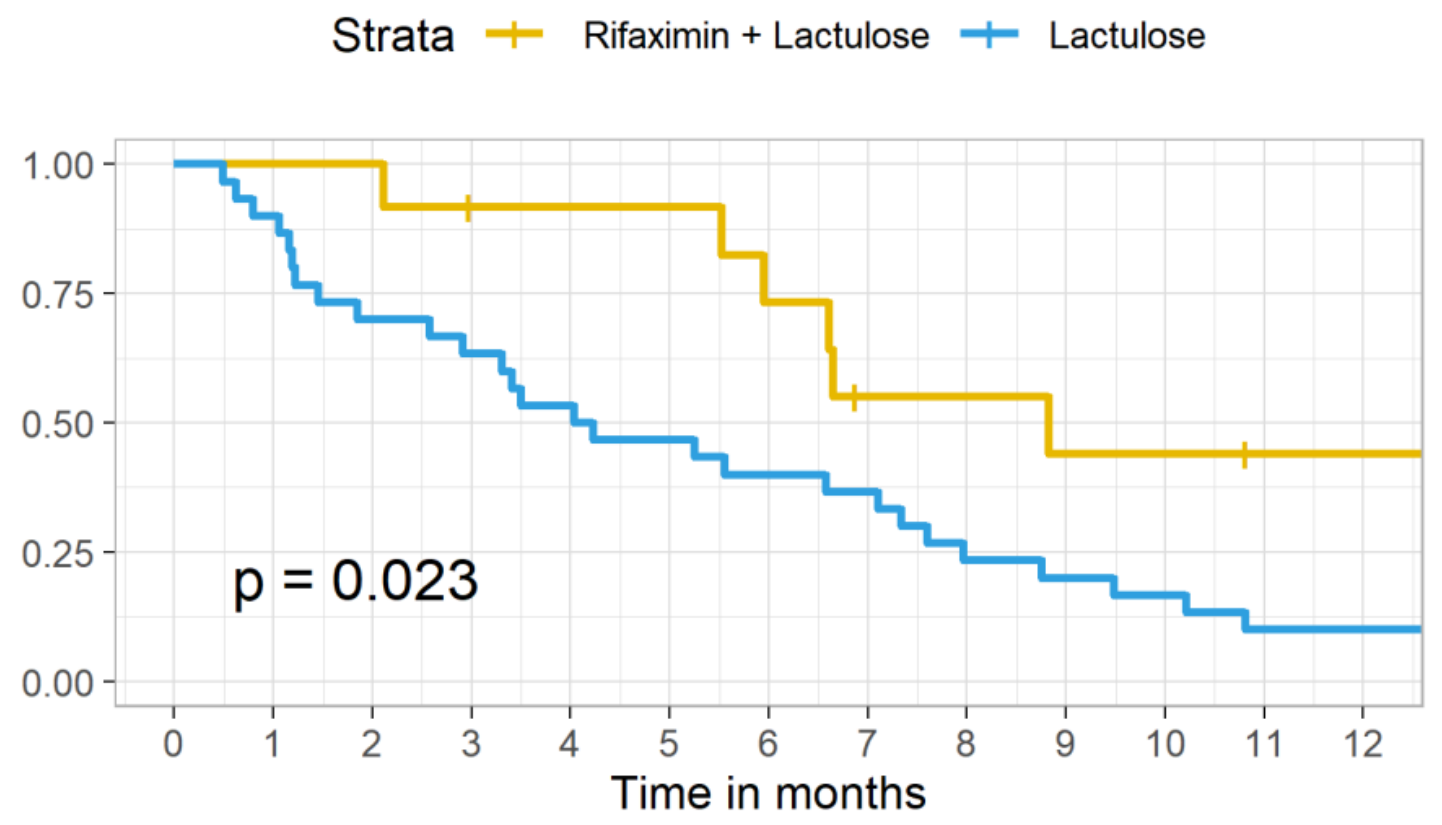

\section{Number at risk}

\begin{tabular}{c|cccccccccccccc|} 
Rifaximin + Lactulose & 12 & 12 & 12 & 10 & 10 & 10 & 8 & 5 & 5 & 4 & 4 & 3 & 3 \\
Lactulose & 30 & 27 & 21 & 19 & 16 & 14 & 12 & 11 & 7 & 6 & 5 & 3 & 3
\end{tabular}

Figure 2. Kaplan-Meier estimates of the efficacy of rifaximin add-on to lactulose (group 1) vs. lactulose monotherapy (group 2) in preventing HE recurrence within 12 months among patients with cirrhosis complicated by hepatic encephalopathy. Time to first HE episode within 12 months of follow-up: Log-Rank $p=0.023$. 
3.3. Comparisons of Various Clinical and Laboratory Parameters between Group $R+L$ vs. Group $L$ after One-Year Follow-Up

As shown in Table 3, the median number of hospitalizations due to HE during one-year follow-up was significantly lower in group R + L vs. group L [1, (0-2) vs. 3, $(2-4), p<0.001]$. The median days of hospitalization attributed to HE within one-year of follow-up was significantly lower in group $\mathrm{R}+\mathrm{L}$ than that in group L [11, (0-27) vs. 37 (15-97), $p=0.003$ ]. $\mathrm{HE}$ recurrence during one-year follow-up was significantly lower in group $\mathrm{R}+\mathrm{L}$ than in group L. HE grade by the 12 th month was also less severe in group $\mathrm{R}+\mathrm{L}$ than that in group $\mathrm{L}$. In addition, greater reduction in serum ammonia was noted in group $\mathrm{R}+\mathrm{L}$ than that in

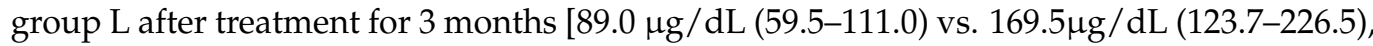

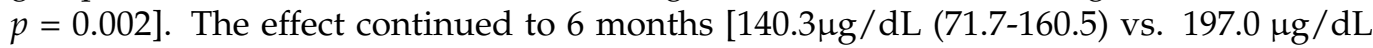
(149.5-304.0), $p=0.007]$. The reduction in ammonia did not maintain to one year.

Table 3. Comparisons of clinical outcomes and laboratory parameters between groups after one-year follow-up.

\begin{tabular}{|c|c|c|c|}
\hline Clinical Parameters & Group 1 Rifaximin + Lactulose & Group 2 Lactulose & $p$-Value \\
\hline Number of hospitalizations due to HE & $1(0.0,2.0)$ & $3(2.0,4.0)$ & $<0.001$ \\
\hline Days of hospitalizations & $11(0.0,27.0)$ & $37(15.0,97.0)$ & 0.003 \\
\hline HE Recurrence & $6(50 \%)$ & $27(87.1 \%)$ & 0.011 \\
\hline HE grade g $^{\$}(\mathrm{n})$ & 7 & 23 & 0.003 \\
\hline Minimal/I/II/III/IV / V: & $1 / 0 / 0 / 1 / 0 / 5$ & $9 / 2 / 6 / 4 / 2 / 0$ & \\
\hline Death $(n, \%)$ & $2(20 \%)$ & $13(41.9 \%)$ & 0.216 \\
\hline MMSE & $25.0 \pm 4.1$ & & \\
\hline $\operatorname{EVB}(n, \%)$ & $6(50 \%)$ & $31(100 \%)$ & 0.313 \\
\hline Ascites $(\mathrm{n})$ & 7 & 21 & 0.307 \\
\hline Nil/Mild/moderate/Severe: & $5 / 1 / 0 / 1$ & $10 / 5 / 1 / 5$ & \\
\hline \multicolumn{4}{|l|}{ Laboratory parameters } \\
\hline Serum Ammonia & $132.2(62.0,222.0)$ & $201(157.0,239.0)$ & 0.179 \\
\hline MELD & $14.6(12.0,20.1)$ & $20(15.0,22.0)$ & 0.115 \\
\hline INR & $1.4(1.4,1.5)$ & $1.5(1.3,1.9)$ & 0.616 \\
\hline $\mathrm{WBC}\left(10^{3} / \mathrm{uL}\right)$ & $4.1(3.6,6.2)$ & $4.3(2.9,8.1)$ & 0.772 \\
\hline Hemoglobin (g/dL) & $65(47.0,94.0)$ & $78(49.0,127.0)$ & 0.825 \\
\hline $\operatorname{PLT}\left(10^{3} / \mathrm{uL}\right)$ & $9.5(8.9,11.5)$ & $9.85(8.5,10.8)$ & 0.386 \\
\hline Creatinine (mg/dL) & $1.165(0.8,1.8)$ & $1.39(0.9,3.4)$ & 0.662 \\
\hline Bilirubin Total (mg/dL) & $2.6(1.0,3.0)$ & $1.9(1.0,4.5)$ & 0.934 \\
\hline AST $(\mathrm{U} / \mathrm{L})$ & $43(29.0,47.0)$ & $48(36.0,55.0)$ & 0.137 \\
\hline $\operatorname{ALT}(\mathrm{U} / \mathrm{L})$ & $26(19.0,46.0)$ & $26(21.0,37.0)$ & 1.0 \\
\hline Albumin (g/dL) & $2.805(2.6,3.0)$ & $2.94(2.6,3.4)$ & 0.861 \\
\hline
\end{tabular}

\$ West-Heaven classification [Conn H.O., Liberathal M.M. The hepatic coma syndromes and lactulose. Williams and Wilkins 1979; 1-121 [DOI:10.1016/0016-5085(79)90191-4].

\subsection{Outcomes Analysis before and after Rifaximin Add-On within Group $R+L$}

In addition, these clinical and laboratory parameters were also compared before and after rifaximin therapy within the group $\mathrm{R}+\mathrm{L}$.

For patients in group $\mathrm{R}+\mathrm{L}$, compared to baseline condition, significant improvement in clinical and laboratory parameters were noticed after rifaximin add on. As shown in Table 4 and Figure 3, the MMSE was improved both at 6 months and 12 months $(p=0.020)$. The number of hospitalizations attributed to $\mathrm{HE}$ also significantly decreased after rifaximin use $(p=0.049)$. The reduction in days of hospitalization attributed to HE significantly decreased at 6 months $(p=0.028)$ and lasted to 12 months $(p=0.046)$. The number of patients who suffered from EVB were also decreased significantly both at 6 and 12 months $(p=0.011)$. Moreover, serum ammonia levels also significantly decreased at 3 and 6 months ( $p=0.0025$ and $p=0.0085$, respectively). 
Table 4. Comparisons of clinical and laboratory parameters at baseline and after rifaximin plus lactulose initiated in patients $(\mathrm{N}=12)$ during different follow-up visits.

\begin{tabular}{ccccc}
\hline & Baseline & 6 Months & 12 Months & $p$-Value \\
\hline Clinical parameters & & & & \\
MMSE & $21.4 \pm 2.2$ & $27.4 \pm 1.1$ & $25.0 \pm 1.8$ & 0.02 \\
EVB & $50 \%$ & $0 \%$ & $0 \%$ & 0.011 \\
Ascites & $41.70 \%$ & $36.40 \%$ & $28.60 \%$ & 0.794 \\
Lab parameters & & & & \\
MELD & $15.0 \pm 1.6$ & $14.9 \pm 1.8$ & $16.1 \pm 1.6$ & 0.524 \\
INR & $1.4 \pm 0.03$ & $1.4 \pm 0.03$ & $1.4 \pm 0.05$ & 0.484 \\
WBC & $4.0 \pm 0.5$ & $4.7 \pm 0.8$ & $4.8 \pm 0.5$ & 0.352 \\
Hb & $9.8 \pm 0.4$ & $10.0 \pm 0.5$ & $9.9 \pm 0.5$ & 0.909 \\
Plt & $71.1 \pm 10.8$ & $79.0 \pm 9.9$ & $74.1 \pm 12.4$ & 0.566 \\
Cr & $2.3 \pm 1.2$ & $1.8 \pm 1.0$ & $3.0 \pm 1.9$ & 0.273 \\
Bilt & $1.8 \pm 0.4$ & $2.2 \pm 0.4$ & $2.2 \pm 0.5$ & 0.268 \\
Alb & $3.0 \pm 0.2$ & $3.0 \pm 0.2$ & $2.9 \pm 0.1$ & 0.45 \\
Serum Ammonia & $248.9 \pm 47.0$ & $133.1 \pm 33.0$ & $176.5 \pm 61.6$ & $0.187^{\dagger}$
\end{tabular}

† $p$-value of serum ammonia by repeated measure ANOVA uses 4 time-points including at 3 months with mean and SD of $108.0 \pm 14.4$. Two patients took rifaximin for less than 12 months, and one for less than 6 months.

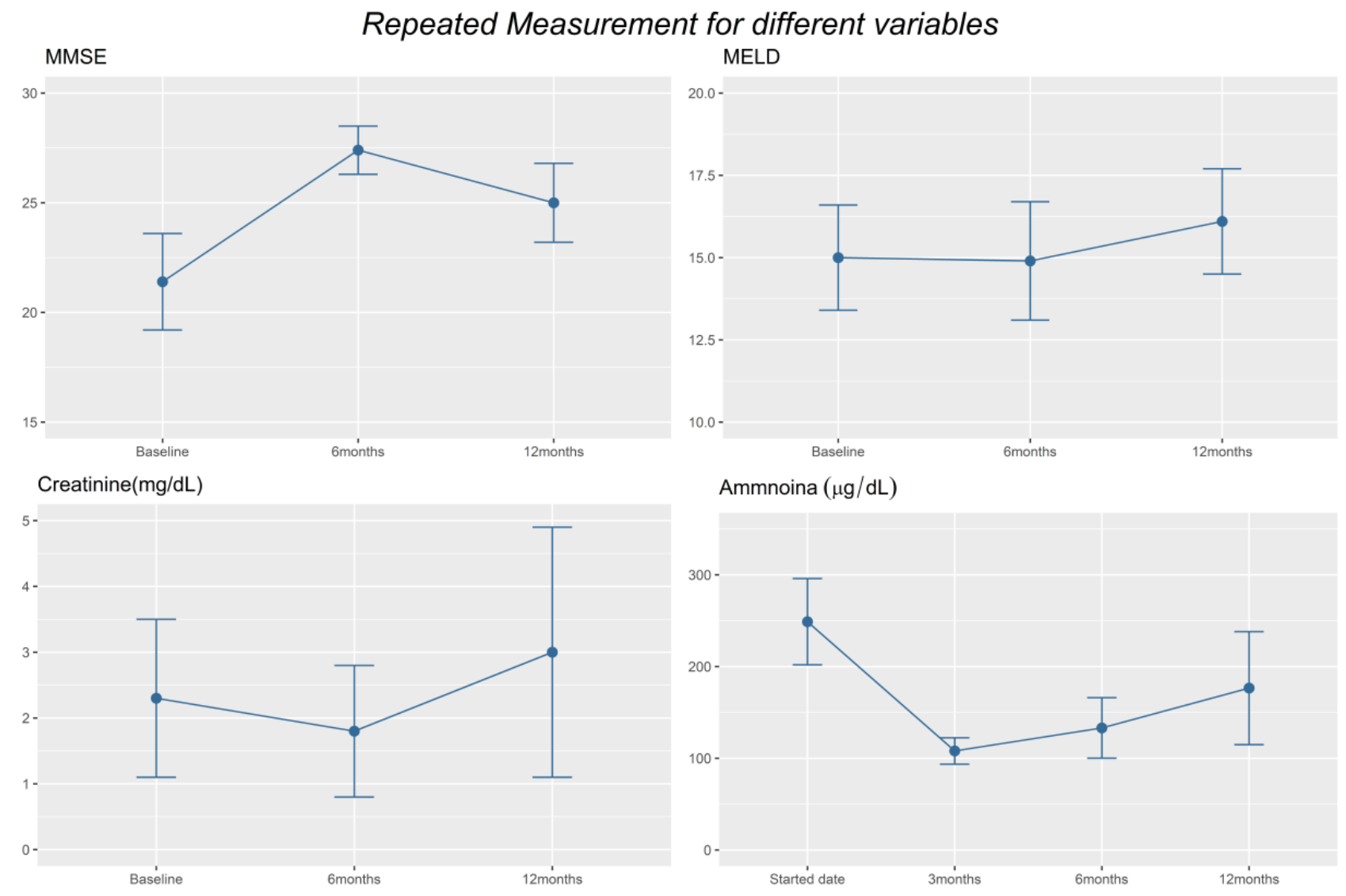

Figure 3. Cont. 

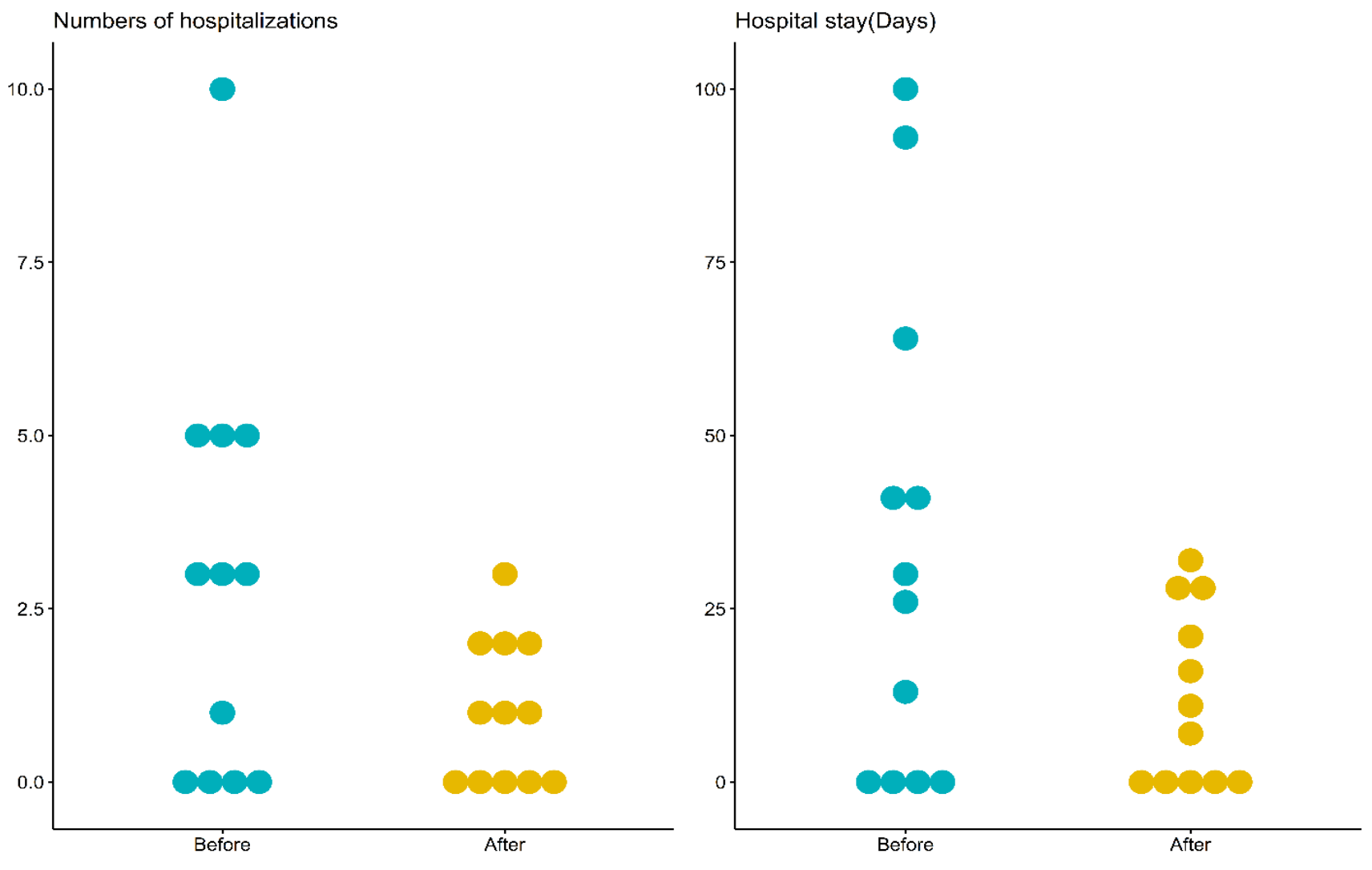

Percentage of Ascites status
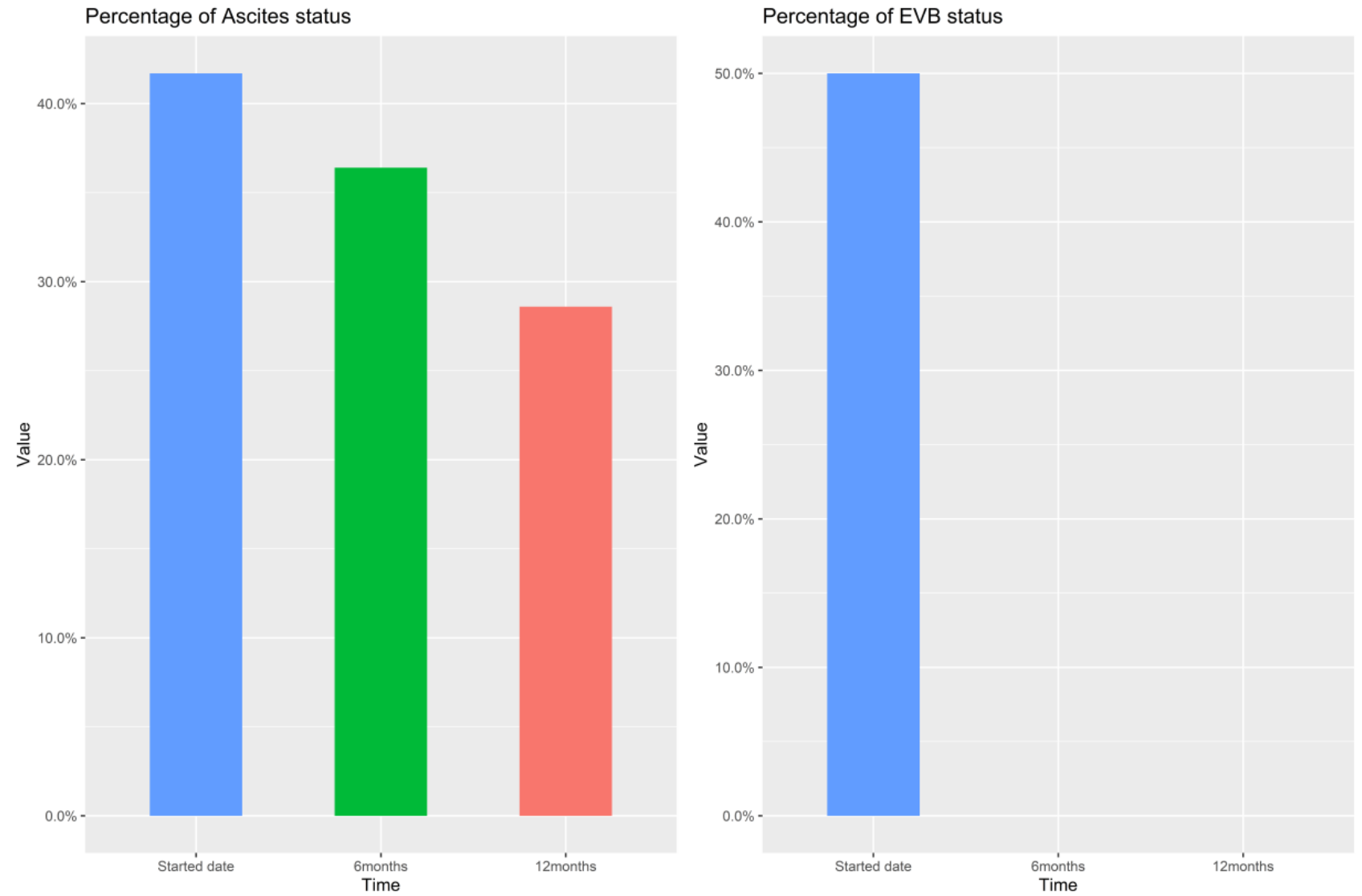

Figure 3. Display of important clinical and laboratory parameter changes in Group 1 patients during different follow-up time points. 


\subsection{Side Effects of Rifaximin}

There was no rifaximin side effects or safety complications recorded in our study.

\section{Discussion}

In this retrospective cohort study, we revealed superior treatment efficacy with rifaximin add-on to lactulose over lactulose alone in patients with cirrhosis complicated by HE at a single medical center in Taiwan. Compared with lactulose monotherapy, we found significant improvement in the maintenance of HE remission and decreased episodes and days of HE-related hospitalizations after add-on rifaximin. The serum ammonia levels were also significantly lower at the 3rd and 6th month in the add-on group. Regarding changes before and after rifaximin add-on in the same patient group, MMSE, episodes of hospitalizations, and number of patients with EVB also improved both at 6 and 12 months. The days of hospitalization and serum ammonia levels at the 3rd and 6th month were also improved. No patient discontinued rifaximin due to adverse events or complications. The above results provide a real-world evidence for the use of rifaximin add-on to lactulose instead of lactulose alone for patients with cirrhosis complicated with hepatic encephalopathy in Taiwan. Comparing the economic burden from rifaximin and the average hospital expense from one of the cirrhotic complications such as hepatic encephalopathy, variceal bleeding and ascites per patient per year in Taiwan [17-19], the economic burden from rifaximin is more cost-effective. Therefore, these results may justify the use and coverage of rifaximin by the National Health Insurance of Taiwan in the future.

Although the comprehensive mechanisms underlying HE are incompletely understood [2], pseudo-neurotransmitters such as gamma-aminobutyric acid (GABA) and ammonia are the best characterized neurotoxins that precipitate HE [20]. It is hypothesized that neurotoxins like ammonia, which is the product of protein metabolized by colonic bacteria and enterocyte, entered systemic circulation via portal vein and across the blood-brain barrier under cirrhotic condition, resulting in neurologic dysfunction, even HE $[2,21]$. The gastrointestinal tract is the primary source of ammonia while gut microbiota is increasingly recognized as another important source of ammonia. Strategies aimed to lower plasma ammonia level and improve gut dysbiosis, such as lactulose and rifaximin, are currently the mainstay of drug therapy for HE. TIPS and orthotopic liver transplantation (OLT) are recognized as the second-line treatment [20]. Other therapies under investigation include L-ornithine-L-aspartate which stimulates the metabolism of ammonia [22], polyethylene glycol (PEG) that helps the excretion of ammonia from stool by cathartic effect [23], and branched chain amino acid supplement that decreases the ratio of plasma aromatic amino acids to branched-chain amino acids [24]. In addition, probiotics containing lactobacilli and bifidobacterial etc., [25], fecal microbiota transplant (FMT) that correct microbial dysbiosis [26], are treatments that require more studies. In addition, the extracorporeal albumin dialysis (ECAD) such as molecular adsorbents recirculating system (MARS) that directly reduce endogenous toxin in case of severe $\mathrm{HE}[27,28]$ is served as a bridging therapy for those planned for OLT. Of note is that FMT may be the rising star in the management of $\mathrm{HE}$ in the future [26,29]. Since the mainstream of drug treatment for HE is lactulose and/or rifaximin [1], the latter is not routinely used in Asia including Taiwan, we therefore conducted the current study to focus on their comparison.

Preventing HE recurrence can avoid heavy burden not only to patients and their families, but also to the healthcare system and society in general [30]. HE-related hospitalization costs are still rising and have increased from $\$ 4.68$ billion in 2005 to $\$ 7.25$ billion in 2009 in the United States [31]. In a systematic review, rifaximin was shown to be associated with shorter hospital stays, reduced healthcare costs, and better cost-effectiveness [32]. In the current AASLD/EASL practice guideline, lactulose is recommended as a first-line pharmacologic agent for preventing recurrent $\mathrm{HE}$, and rifaximin is suggested as an add-on therapy if recurrence of HE persists [1]. Kang et al. discovered that rifaximin combined with lactulose was superior to lactulose monotherapy in preventing recurrent $\mathrm{HE}$ in nonHCC patients [13]. However, one randomized controlled trial (RCT) found there was no 
difference between rifaximin and placebo in maintaining HE remission [12]. Another study found rifaximin combined with lactulose to be non-superior to lactulose monotherapy in treatment of refractory HE [14]. Obviously, the role of adding rifaximin in preventing HE recurrence is still a controversial issue. However, our study demonstrated that time to first $\mathrm{HE}$ recurrence was significantly longer in the rifaximin add-on group than the lactulose monotherapy group, which is a consequential finding [10]. The odds ratio, which reflects the risk of $\mathrm{HE}$ recurrence, was also significantly lower in patients receiving rifaximin add-on therapy. There appears to be additional benefit of rifaximin in the prevention of recurrent HE. Furthermore, previous studies have demonstrated the effect of rifaximin in reducing HE-related hospitalization $[9,10,33]$, and our study further confirmed a decrease in number of episodes and days of hospitalization among patients receiving rifaximin and lactulose combination therapy compared to lactulose monotherapy, suggesting better cost-effectiveness (median decrease in days of hospitalizations from 37 to 11 days). In addition, there was greater reduction in serum ammonia level in the combination therapy group compared to the monotherapy group.

As we know, the development of complications from cirrhosis, such as ascites, variceal bleeding, and HE, correlates with progressively severe portal hypertension [34]. Studies have demonstrated the efficacy of rifaximin in reducing cirrhosis-related complications [13,35], and our study also reveals improvement following long-term, such as a decrease in esophageal variceal bleeding potentially linked to decreased portal hypertension.

MMSE is a widely used tool for cognitive function assessment. Although precise utility in evaluating HE is unclear, many studies employ the MMSE to evaluate cognition in cirrhotic patients with HE [36-39]. Our study also demonstrated cognitive dysfunction improvement by MMSE significantly after rifaximin add-on therapy for six months.

No patients discontinued rifaximin therapy due to adverse effects in our study. Rifaximin is safe and well-tolerated for long-term use, as is consistent with the previous reports $[9,11]$. Neff et al. considered rifaximin as a more cost-effective agent for HE treatment due to its efficacy on HE prevention despite its high price [40].

There are some limitations of our study. First, only a few patients were enrolled in the rifaximin add-on group, and age, HE grade, HCC status, and MELD score at baseline were not matched to the control group. However, a multivariable logistic regression analysis had been performed to adjust for these confounding factors and the result can provide evidence for conducting a larger prospective matched study in the future. Second, because of missing MMSE scores in the lactulose monotherapy group, we could not evaluate whether there was a difference in MMSE between these two groups after therapy. Third, due to retrospective design, we did not evaluate the therapeutic effect of rifaximin on the changes of sarcopenia, quality of life, or small intestine bacterial overgrowth.

In conclusion, the results of our study demonstrate that one-year efficacy of rifaximin add-on to lactulose is safe, well tolerated, and superior to lactulose alone in reducing HE recurrence and HE-related hospitalization in one-year follow-up in Taiwan. A large-scale prospective randomized control study may be warranted.

Author Contributions: Conceptualization, C.-H.H. and R.-N.C.; methodology, H.-J.T.; software, H.-J.T. and F.-C.Y.; validation, H.-J.T. and F.-C.Y.; formal analysis, H.-J.T.; investigation, H.-J.T. and F.-C.Y.; resources, C.-H.H. and R-N.C.; data curation, C.C. and C.-H.H.; writing-original draft preparation, C.C.; writing-review and editing, C.-H.H. and R.-N.C.; visualization, supervision, R.-N.C.; project administration, C.-H.H. and R.-N.C.; funding acquisition, C.-H.H. and R.-N.C. All authors have read and agreed to the published version of the manuscript.

Funding: This research was funded by the Chang Gung Medical Research Projects: CMRPG3K2191; National Science Council: NMRPG3K0261.

Institutional Review Board Statement: The study was conducted according to the guidelines of the Declaration of Helsinki, and approved by the Institutional Review Board of Linkou Chang Gung Memorial Hospital (protocol code 201701810B0). 
Informed Consent Statement: Informed consent was obtained from all subjects involved in the study. Written informed consent has been obtained from the patients to publish this paper.

Data Availability Statement: Data will be available under readers request if indicated.

Conflicts of Interest: The authors declare no conflict of interest.

\section{References}

1. Vilstrup, H.; Amodio, P.; Bajaj, J.; Cordoba, J.; Ferenci, P.; Mullen, K.D.; Weissenborn, K.; Wong, P. Hepatic encephalopathy in chronic liver disease: 2014 Practice Guideline by the American Association for the Study of Liver Diseases and the European Association for the Study of the Liver. Hepatology 2014, 60, 715-735. [CrossRef]

2. Wijdicks, E.F. Hepatic Encephalopathy. N. Engl. J. Med. 2016, 375, 1660-1670. [CrossRef]

3. Montagnese, S.; Bajaj, J.S. Impact of Hepatic Encephalopathy in Cirrhosis on Quality-of-Life Issues. Drugs 2019, 79, 11-16. [CrossRef]

4. Elwir, S.; Rahimi, R.S. Hepatic Encephalopathy: An Update on the Pathophysiology and Therapeutic Options. J. Clin. Transl. Hepatol. 2017, 5, 142-151. [CrossRef]

5. Sharma, B.C.; Sharma, P.; Agrawal, A.; Sarin, S.K. Secondary prophylaxis of hepatic encephalopathy: An open-label randomized controlled trial of lactulose versus placebo. Gastroenterology 2009, 137, 885-891.e1. [CrossRef]

6. Liu, A.; Perumpail, R.B.; Kumari, R.; Younossi, Z.M.; Wong, R.J.; Ahmed, A. Advances in cirrhosis: Optimizing the management of hepatic encephalopathy. World J. Hepatol. 2015, 7, 2871-2879. [CrossRef]

7. Phongsamran, P.V.; Kim, J.W.; Cupo Abbott, J.; Rosenblatt, A. Pharmacotherapy for hepatic encephalopathy. Drugs 2010, 70, 1131-1148. [CrossRef]

8. Scarpignato, C.; Pelosini, I. Rifaximin, a poorly absorbed antibiotic: Pharmacology and clinical potential. Chemotherapy 2005, 51, 36-66. [CrossRef]

9. Mullen, K.D.; Sanyal, A.J.; Bass, N.M.; Poordad, F.F.; Sheikh, M.Y.; Frederick, R.T.; Bortey, E.; Forbes, W.P. Rifaximin is safe and well tolerated for long-term maintenance of remission from overt hepatic encephalopathy. Clin. Gastroenterol. Hepatol. 2014, 12, 1390-1397.e2. [CrossRef]

10. Bass, N.M.; Mullen, K.D.; Sanyal, A.; Poordad, F.; Neff, G.; Leevy, C.B.; Sigal, S.; Sheikh, M.Y.; Beavers, K.; Frederick, T.; et al. Rifaximin treatment in hepatic encephalopathy. N. Engl. J. Med. 2010, 362, 1071-1081. [CrossRef]

11. Mas, A.; Rodes, J.; Sunyer, L.; Rodrigo, L.; Planas, R.; Vargas, V.; Castells, L.; Rodriguez-Martinez, D.; Fernandez-Rodriguez, C.; Coll, I.; et al. Comparison of rifaximin and lactitol in the treatment of acute hepatic encephalopathy: Results of a randomized, double-blind, double-dummy, controlled clinical trial. J. Hepatol. 2003, 38, 51-58. [CrossRef]

12. Ali, B.; Zaidi, Y.A.; Alam, A.; Anjum, H.S. Efficacy of Rifaximin in prevention of recurrence of hepatic encephalopathy in patients with cirrhosis of liver. J. Coll. Physicians Surg. Pak. 2014, 24, 269-273. Available online: https://www.jcpsp.pk/archive/2014 / Apr2014/13.pdf (accessed on 27 May 2021).

13. Kang, S.H.; Lee, Y.B.; Lee, J.H.; Nam, J.Y.; Chang, Y.; Cho, H.; Yoo, J.J.; Cho, Y.Y.; Cho, E.J.; Yu, S.J.; et al. Rifaximin treatment is associated with reduced risk of cirrhotic complications and prolonged overall survival in patients experiencing hepatic encephalopathy. Aliment. Pharmacol. Ther. 2017, 46, 845-855. [CrossRef] [PubMed]

14. Ahire, K.; Sonawale, A. Comparison of Rifaximin Plus Lactulose with the Lactulose Alone for the Treatment of Hepatic Encephalopathy. J. Assoc. Physicians India 2017, 65, 42-46. [PubMed]

15. Courson, A.; Jones, G.M.; Twilla, J.D. Treatment of Acute Hepatic Encephalopathy: Comparing the Effects of Adding Rifaximin to Lactulose on Patient Outcomes. J. Pharm. Pract. 2016, 29, 212-217. [CrossRef] [PubMed]

16. Vlachogiannakos, J.; Viazis, N.; Vasianopoulou, P.; Vafiadis, I.; Karamanolis, D.G.; Ladas, S.D. Long-term administration of rifaximin improves the prognosis of patients with decompensated alcoholic cirrhosis. J. Gastroenterol. Hepatol. 2013, 28, 450-455. [CrossRef]

17. Hsieh, C.R.; Kuo, C.W. Cost of chronic hepatitis B virus infection in Taiwan. J. Clin. Gastroenterol. 2004, 38, S148-S152. [CrossRef]

18. Lin, W.A.; Tarn, Y.H.; Tang, S.L. Cost-utility analysis of different peg-interferon alpha-2b plus ribavirin treatment strategies as initial therapy for naive Chinese patients with chronic hepatitis C. Aliment. Pharmacol. Ther. 2006, 24, 1483-1493. [CrossRef]

19. El Khoury, A.C.; Wallace, C.; Klimack, W.K.; Razavi, H. Economic burden of hepatitis C-associated diseases: Europe, Asia Pacific, and the Americas. J. Med. Econ. 2012, 15, 887-896. [CrossRef]

20. Aldridge, D.R.; Tranah, E.J.; Shawcross, D.L. Pathogenesis of hepatic encephalopathy: Role of ammonia and systemic inflammation. J. Clin. Exp. Hepatol. 2015, 5, S7-S20. [CrossRef]

21. Hadjihambi, A.; Arias, N.; Sheikh, M.; Jalan, R. Hepatic encephalopathy: A critical current review. Hepatol. Int. 2018, 12, 135-147. [CrossRef]

22. Sidhu, S.S.; Sharma, B.C.; Goyal, O.; Kishore, H.; Kaur, N. L-ornithine L-aspartate in bouts of overt hepatic encephalopathy. Hepatology 2018, 67, 700-710. [CrossRef]

23. Rahimi, R.S.; Singal, A.G.; Cuthbert, J.A.; Rockey, D.C. Lactulose vs polyethylene glycol 3350-electrolyte solution for treatment of overt hepatic encephalopathy: The HELP randomized clinical trial. JAMA Intern. Med. 2014, 174, 1727-1733. [CrossRef] 
24. Les, I.; Doval, E.; Garcia-Martinez, R.; Planas, M.; Cardenas, G.; Gomez, P.; Flavia, M.; Jacas, C.; Minguez, B.; Vergara, M.; et al. Effects of branched-chain amino acids supplementation in patients with cirrhosis and a previous episode of hepatic encephalopathy: A randomized study. Am. J. Gastroenterol. 2011, 106, 1081-1088. [CrossRef]

25. Agrawal, A.; Sharma, B.C.; Sharma, P.; Sarin, S.K. Secondary prophylaxis of hepatic encephalopathy in cirrhosis: An open-label, randomized controlled trial of lactulose, probiotics, and no therapy. Am. J. Gastroenterol. 2012, 107, 1043-1050. [CrossRef]

26. Bajaj, J.S.; Kassam, Z.; Fagan, A.; Gavis, E.A.; Liu, E.; Cox, I.J.; Kheradman, R.; Heuman, D.; Wang, J.; Gurry, T.; et al. Fecal microbiota transplant from a rational stool donor improves hepatic encephalopathy: A randomized clinical trial. Hepatology 2017, 66, 1727-1738. [CrossRef]

27. Hassanein, T.I.; Tofteng, F.; Brown, R.S., Jr.; McGuire, B.; Lynch, P.; Mehta, R.; Larsen, F.S.; Gornbein, J.; Stange, J.; Blei, A.T. Randomized controlled study of extracorporeal albumin dialysis for hepatic encephalopathy in advanced cirrhosis. Hepatology 2007, 46, 1853-1862. [CrossRef]

28. Banares, R.; Nevens, F.; Larsen, F.S.; Jalan, R.; Albillos, A.; Dollinger, M.; Saliba, F.; Sauerbruch, T.; Klammt, S.; Ockenga, J.; et al. Extracorporeal albumin dialysis with the molecular adsorbent recirculating system in acute-on-chronic liver failure: The RELIEF trial. Hepatology 2013, 57, 1153-1162. [CrossRef] [PubMed]

29. Bajaj, J.S.; Gavis, E.A.; Fagan, A.; Wade, J.B.; Thacker, L.R.; Fuchs, M.; Patel, S.; Davis, B.; Meador, J.; Puri, P.; et al. A Randomized Clinical Trial of Fecal Microbiota Transplant for Alcohol Use Disorder. Hepatology 2021, 73, 1688-1700. [CrossRef]

30. Poordad, F.F. Review article: The burden of hepatic encephalopathy. Aliment. Pharmacol. Ther. 2007, 25, 3-9. [CrossRef]

31. Flamm, S.L. Considerations for the cost-effective management of hepatic encephalopathy. Am. J. Manag. Care 2018, 24, S51-S61.

32. Neff, G.; Zachry, W., III. Systematic Review of the Economic Burden of Overt Hepatic Encephalopathy and Pharmacoeconomic Impact of Rifaximin. Pharmacoeconomics 2018, 36, 809-822. [CrossRef]

33. Neff, G.W.; Kemmer, N.; Zacharias, V.C.; Kaiser, T.; Duncan, C.; McHenry, R.; Jonas, M.; Novick, D.; Williamson, C.; Hess, K.; et al. Analysis of hospitalizations comparing rifaximin versus lactulose in the management of hepatic encephalopathy. Transplant. Proc. 2006, 38, 3552-3555. [CrossRef] [PubMed]

34. D'Amico, G.; Morabito, A.; D’Amico, M.; Pasta, L.; Malizia, G.; Rebora, P.; Valsecchi, M.G. Clinical states of cirrhosis and competing risks. J. Hepatol. 2018, 68, 563-576. [CrossRef] [PubMed]

35. Salehi, S.; Tranah, T.H.; Lim, S.; Heaton, N.; Heneghan, M.; Aluvihare, V.; Patel, V.C.; Shawcross, D.L. Rifaximin reduces the incidence of spontaneous bacterial peritonitis, variceal bleeding and all-cause admissions in patients on the liver transplant waiting list. Aliment. Pharmacol. Ther. 2019, 50, 435-441. [CrossRef] [PubMed]

36. Yoo, H.Y.; Edwin, D.; Thuluvath, P.J. Relationship of the model for end-stage liver disease (MELD) scale to hepatic encephalopathy, as defined by electroencephalography and neuropsychometric testing, and ascites. Am. J. Gastroenterol. 2003, 98, 1395-1399. [CrossRef]

37. Malaguarnera, M.; Gargante, M.P.; Cristaldi, E.; Vacante, M.; Risino, C.; Cammalleri, L.; Pennisi, G.; Rampello, L. Acetyl-Lcarnitine treatment in minimal hepatic encephalopathy. Dig. Dis. Sci. 2008, 53, 3018-3025. [CrossRef]

38. Koziarska, D.; Wunsch, E.; Milkiewicz, M.; Wojcicki, M.; Nowacki, P.; Milkiewicz, P. Mini-Mental State Examination in patients with hepatic encephalopathy and liver cirrhosis: A prospective, quantified electroencephalography study. BMC Gastroenterol. 2013, 13, 107. [CrossRef]

39. Corrias, M.; Turco, M.; Rui, M.D.; Gatta, A.; Angeli, P.; Merkel, C.; Amodio, P.; Schiff, S.; Montagnese, S. Covert hepatic encephalopathy: Does the mini-mental state examination help? J. Clin. Exp. Hepatol. 2014, 4, 89-93. [CrossRef]

40. Neff, G.W.; Kemmer, N.; Duncan, C.; Alsina, A. Update on the management of cirrhosis-Focus on cost-effective preventative strategies. Clinicoecon. Outcomes Res. 2013, 5, 143-152. [CrossRef] 